\title{
Business intelligence, big data and theory
}

Again, the articles for this issue are mostly about the application of new technology and about business intelligence, reflecting a strong development in corporations. The only exception is the first article, which is purely theoretical.

The contribution by Søilen, entitled "When the social sciences are based in evolutionary theory: the example of geoeconomics and intelligence studies," is a theoretical article. It argues for why it was wrong to make the study of physics the model for the new social sciences after the Second World War. Moreover, it describes how this was done for the study of economics and how new studies like geoeconomics and intelligence studies have an advantage in this sense, and that a fresh look at theory is easier in these cases.

Hughes, in his article "A new model for identifying emerging technologies," argues for the relevance of the intelligence expert despite the increase in new complexities required for understanding an industry, but he also emphasizes the importance for the analyst to learn more about big data. Our technological systems are still ineffective at knowing the relevant data sources and how to connect the data in meaningful ways to derive value for the firm, but their importance is increasing. The author proposes a new forecasting model that incorporates a combination of technology sequencing analysis and big data tools within the organization while also leveraging experts from across the open innovation spectrum.

Salguero et al., in the article "Proposal of an assessment scale in competitive intelligence applied to touristic sector," present a mathematical CI model to be applied in the tourism sector, specifically for hotels. The model is also tested and fine-tuned, proving to have value for the CI function. The authors also present an extensive literature review.

The extensive article by García and Pinzón, "Key success factors to business intelligence solutions implementation," builds on previous literature published in this journal, such as Cidrin and Adamala (2011) and takes as a starting point the high number of BI projects that fail. The authors identify 13 factors that affect business intelligence solution success.

The final article, by Papachristodoulou et al., "Business intelligence and SMEs: Bridging the gap," talks at great length about the problem of implementing BI in small and medium enterprises (SMEs). It shows how new products have changed to adapt to a new sector of customers.

On behalf of the Editorial Board,

Sincerely Yours,

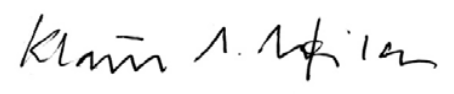

Prof. Dr. Klaus Solberg Søilen Halmstad University, Sweden Editor-in-chief 